\title{
ON THE EQUIVARIANT HOMOTOPY TYPE OF G-ANR'S
}

\author{
SEAWOMIR KWASIK
}

\begin{abstract}
I show that every metric $G$-ANR has the $G$-homotopy type of a $G$-CW complex. Therefore I. James and G. Segal's results concerning equivariant homotopy type are special cases of the Whitehead theorem for $G-C W$ complexes.
\end{abstract}

In this note $G$ is assumed to be a compact Lie group. A metric $G$-space $X$ is said to be a $G$-ANR if for any $G$-embedding $i: X \rightarrow Y$ in a metric $G$-space $Y$ such that $i X$ is closed in $Y$, the image $i X$ is a $G$-retract of some open invariant neighborhood of $Y$.

THEOREM. Every metric G-ANR has the G-homotopy type of a G-CW complex.

As a consequence of this theorem and Theorem 5.3 in [3] we obtain the following.

Corollary (James, Segal [1]). Let $f: X \rightarrow Y$ be a $G$ map between $G$-ANR's. Then $f$ is a G-homotopy equivalence iff $f^{H}: X^{H} \rightarrow Y^{H}$ is an ordinary homotopy equivalence for every closed subgroup $H \subseteq G$.

Proof of Theorem. Let $X$ be a metric $G$-ANR. By the equivariant version of a standard argument (Lemma 4.7 of [4]), it suffices to prove that $X$ is $G$-dominated by a $G-C W$ complex. Observe that every metric $G$-space $X$ may be $G$-embedded as a closed $G$-subset of a convex $G$-set in a Banach space of bounded real-valued functions on $X$ with $G$-action given by $g(h)(x)=h\left(g^{-1}(x)\right)$ for $g \in G, h: X \rightarrow R$, and $x \in X$.

Therefore we may assume that $X$ is a closed $G$-subset of a convex $G$-set $C$ in a Banach $G$-space.

Being a $G$-ANR, $X$ is a $G$-retract of some open neighborhood $U$ of $X$ in $C$; in particular it is $G$-dominated by some $G-C W$ complex. This is seen by an easy modification of the proof of Theorem 3.B in [2]. We obtain that $U$ is $G$-dominated by a $G$-nerve induced by a locally finite refined slice covering and this $G$-nerve has the $G$-homotopy type of a $G-C W$ complex (which is a direct limit of barycentric manifolds in the notation of [2]).

REMARK. Matumoto's proof that a barycentric manifold is a $G-C W$ complex is incorrect because it relies on a result of Yang which relies on an incorrect result of Cairns. A correct proof that smooth manifolds are $G-C W$ complexes is in a preprint Triangulation of stratified fibre bundles by Andrei Verona. The weaker

Received by the editors August 8, 1980.

AMS (MOS) subject classifications (1970). Primary 57E10.

(c) 1981 American Mathematical Society 0002-9939/81/0000-0443/\$01.50 
assertion that smooth $G$-manifolds have the $G$-homotopy type of $G$-CW complexes (which is all that might be needed for this paper is immediate from [4]).

I wish to thank the referee for his helpful suggestions.

\section{REFERENCES}

1. I. M. James and G. B. Segal, On equivariant homotopy type, Topology 17 (1978), 267-272.

2. T. Matumoto, Equivariant $K$-theory and Fredholm operators, J. Fac. Sci. Univ. Tokyo Sect. IA Math. 18 (1971), 109-125.

3. , On G-CW complexes and theorem of J. H. C. Whitehead, J. Fac. Sci. Univ. Tokyo Sect. IA Math. 18 (1971), 363-374.

4. S. Waner, Equivariant homotopy theory and Milnor's theorem, Trans. Amer. Math. Soc. 258 (1980), 351-368.

InSTytut MATEmatyki, UNIWERsytet Gdański, ul Wrta Stwosza 57, 80-952 Gdańsk, Poland 